\title{
Development of strategies to promote healthy sexuality based on Iranian girls perspective about the role of virginity in the construction of their sexuality: an explanatory sequential mixed method study protocol
}

Somayyeh Naghizadeh ${ }^{1}$, Raziyeh Maasoumi ${ }^{1,2^{*}}$ (D) Farideh Khalajabadi-Farahani ${ }^{3}$ and Mojgan Mirghafourvand ${ }^{4}$

\begin{abstract}
Background: The modern Iran is a blend of tradition and modernity, but its dominant culture is still traditional and takes pride in female virginity. On the other hand, the influential factors such as modernity, education, social networks, global communication, influence from advanced Western societies, have obviously led to the emergence of a freer sexual attitude associated with less significance of virginity. Since the traditional, cultural and religious significance of virginity among Iranian girls can shape their sexual behaviors, therefore, the aim of the present study is development of strategies to promote healthy sexuality based on Iranian girls perspective about the role of virginity in the construction of their sexuality.
\end{abstract}

Methods/design: This is a two-stage study; an explanatory sequential mixed-methods follow-up design will be employed in the first stage, which consists of two quantitative and qualitative phases. The first phase is the crosssectional survey that will be conducted on 700 single girls born in the 1970s, 1980s and 1990s. The sample will be selected using the cluster sampling method in the health centers of Tabriz-Iran. The determined sample size will be divided among the selected health centers based on the quota criterion, and eligible households will be selected randomly from the said centers using the SIB website (sib.tbzmed.ac.ir). To collect the required data in the quantitative phase, we will use a researcher-made questionnaire to evaluate the girls' views about virginity and its influential factors, designed based on the theory of "social construction of sexuality". After quantitative data collection and analysis, the findings inform qualitative data collection and analysis. The qualitative phase of the study will be conducted on girls living in Tabriz using in-depth and semi-structured individual interviews and purposive sampling method to collect the required qualitative data. The collected data will be analyzed using the conventional content analysis approach. The findings of two phase will be integrated for further explanation and interpretation to be used in the second stage. In the second phase of this study, a nominal group meeting will be held with the participation

\footnotetext{
*Correspondence: r.masoomie@gmail.com

${ }^{1}$ Department of Midwifery and Reproductive Health, School of Nursing

and Midwifery, Tehran University of Medical Sciences, P.O. Box:

1419733171, Tehran, Iran

Full list of author information is available at the end of the article
}

(c) The Author(s) 2021. Open Access This article is licensed under a Creative Commons Attribution 4.0 International License, which permits use, sharing, adaptation, distribution and reproduction in any medium or format, as long as you give appropriate credit to the original author(s) and the source, provide a link to the Creative Commons licence, and indicate if changes were made. The images or other third party material in this article are included in the article's Creative Commons licence, unless indicated otherwise in a credit line to the material. If material is not included in the article's Creative Commons licence and your intended use is not permitted by statutory regulation or exceeds the permitted use, you will need to obtain permission directly from the copyright holder. To view a copy of this licence, visit http://creativecommons.org/licenses/by/4.0/. The Creative Commons Public Domain Dedication waiver (http://creativeco mmons.org/publicdomain/zero/1.0/) applies to the data made available in this article, unless otherwise stated in a credit line to the data. 
of reproductive and sexual health experts. The strategies extracted from the results of the first phase and review the texts in this meeting will be provided to experts and after receiving the opinions and ideas of the relevant group of experts and prioritizing them, appropriate strategies to improve and promote the healthy sexuality of Iranian girls will be presented.

Discussion: This study is one of the few studies conducted in the field of sexual health and culture in Iran, that using a "mixed-methods" approach to determine and explain the role of virginity in the construction of the sexuality from the Iranian girls perspective. We hope that this study can present evidence-based documents from the latest physical, psychological and social developments in young Iranian girls'sexuality and that the presented healthy sexuality promotion strategies, which will be based on Iranian socio-cultural developments, can provide the basic information required for policy-making and planning for young girls' sexual health. It is also hoped that the findings of this study will be useful in culture-based sexuality education and support for reproductive and sexual health care for the young Iranian generation.

\section{Plain language summary}

Custom, culture and religion in many communities impose virginity on the women of their communities. According to the traditional and conservative Iranian culture, premarital sexual relations are forbidden, female virginity is honor, and it is valuable and necessary for a girl to maintain and take care of her virginity. Attitudes toward sexual relationship and its moral aspects have changed dramatically in many parts of the world in recent decades. Iran, as a conservative society, is no exception to this rule and has experienced substantial social and attitudinal changes over the past decades. This is a two-stage study; an explanatory sequential mixed-methods follow-up design will be employed in the first stage, which consists of two quantitative and qualitative phases. At first quantitative data collection and analysis will be conducted, then the findings inform qualitative data collection and analysis. The findings of two phase will be integrated for further explanation and interpretation to be used in the second stage. In the second stage of the study, the researchers will develop appropriate strategies for the girls' sexual health promotion by a combination of the relevant experts'views obtained in the nominal group meeting and the results extracted from a review of literature as well as the results of the quantitative and qualitative phases of the first stage. it seems that the present study, by employing a better quantitative and qualitative approach, can clarify the young generation's view in the sociocultural background of Iran and explore the possible inconsistency and contradictions in the modern Iranian society to determine whether the traditional view of the concept of virginity in Iran is still dominant, or it has been changed by factors such as modernization, global communications, influence from advanced Western societies.

Keywords: Virginity, Sexuality, Iranian girls, Healthy sexuality

\section{Background}

"Virginity" has a French root in which "Vir" means "man" and "Genere" means "created for". The term has its roots in medieval Western culture and refers to the ownership of a man over a woman [1], and was used for a woman who has never had a sexual relationship with a man $[2,3]$. However, the participants of a study conducted by Carpenter in 2002 identified penile-vaginal intercourse as the act denoting their virginity loss [4].

Virginity is a complex social concept $[4,5]$ with different definitions for people with different cultural backgrounds [6]. In advanced societies such as the United States, sexual activity among adolescents and youth is customary and considered the norm. In fact, changes in social and sexual norms in these societies have caused most people to have pre-marital sexual relationships (men 92.2\% and women 91.9\% [7]). In these societies, males are less inclined to partner with virgin females, and virgins are not considered good sexual partners $[1$, 8-10].

However, in traditional patriarchal societies (where gender roles are clearly defined and very rigidly executed), a woman is important not only in terms of fertility and personality, but also in terms of virginity [11]. In many countries such as Zimbabwe [12], Zambia [13], Ethiopia [14], Ghana [2], Democratic Republic of the Congo [15], Vietnam [16, 17], India [18], Philippines [19], Thailand [20], Indonesia [21], Lebanon [22] and other traditional societies, cultural norms and social unrest affect sexual relations, and maintaining virginity until marriage is common and mandatory so that the hymen is considered socio-culturally a sign of females' chastity and righteousness [23].

Custom, culture and religion create an image of women who impose "female virginity" on their communities [15]. Attitudes toward sexual relationship and its moral 
aspects have changed dramatically in many parts of the world in recent decades [24, 25]. Iran, as a conservative society, has experienced substantial social and attitudinal changes over the past decades [16-22].

The modern Iran is a blend of tradition and modernity, but its dominant culture is still traditional and takes pride in female virginity [26]. According to the traditional and conservative Iranian culture, premarital sexual relations are forbidden [27], female virginity is a honor, and it is valuable and necessary for a girl to maintain and take care of her virginity. Any damage to a girl's hymen prior to marriage would mean great catastrophe and can have dire consequences for her and her family [28]. Versus, the increasingly large young population of Iran and its great influence from international developments, especially in the age of communication, and the modernization of social relations have laid the foundation for converting tradition to modernity [29].

With the development of modernity and modernism in Iranian society, the status of marriage has changed and has increased the age of marriage or celibacy, delay in marriage has increased extramarital relationships and sexual desire disorders [30]. Farahani et al. (2018) carried out a study on university girls and showed that one of the most important considerations for girls and even boys in sexual relations is to preserve their virginity [31]. However, there is evidence that premarital sexual behavior is on the rise among Iranian youth, especially in large cities, but it is often hidden with unknown details [27, 32-36].

In Rahmani et al's study (2016), participants cited "custom" as the main reason for the lack of premarital sexual relations and considered physical virginity the only red line for Iranian girls. In fact, those girls who wanted to experience sexual relations said that they were inclined to have any type of sexual relationship, but on the condition that their hymens would remain intact. Although physical virginity can be a deterrent to sexual contact for a significant proportion of single girls, it may be a factor of further vulnerability for another proportion of girls who have non-vaginal sexual experience [36]. Therefore, the lack of a common and reasonable concept of virginity in relation to the type of sexual relation and the dominant perception of virginity loss only through vaginal intercourse lead to an increase in sexually transmitted diseases and HIV [37, 38].

In Iran, between the first years after the Islamic Revolution and the end of the Iran-Iraq war, the issue of "boy-girl relationships" was less of a "social issue" due to people's strong adherence to socio-cultural values and involvement in the war. However, in the 1970s, they gradually developed relationships due to fundamental social changes and the emergence of a new young generation with different values and beliefs. A change of values has led to broader social changes [39]. Therefore, based on the socio-cultural developments in Iran, it seems necessary to study Iranian girls perspective about the concept of virginity.

The importance of virginity can be discussed from different perspectives. On the one hand, it is identified as an important factor in controlling sexually transmitted diseases and unwanted pregnancies among adolescents and youth as a strategy to control the negative consequences of premarital sex [37]. On the other hand, the importance of physical preservation of virginity or the hymen may be associated with an increased prevalence of unprotected non-vaginal sexual intercourse, which puts girls' sexual health at risk [40]. Therefore, virginity is closely related to sexual health and fertility and this issue should be investigated in more depth with regard to sexual health and fertility.

There is a kind of inconsistency and contradiction in understanding the concept of virginity in the modern Iranian society. Although the cultural and social values of this society emphasize the preservation of virginity, influential factors such as modernity, education, social networks, global communication, influence from advanced Western societies, have obviously led to the emergence of a freer sexual attitude associated with less significance of virginity [41, 42]. Therefore, since Iranian society is a blend of tradition and modernity, it can be an excellent context for a study on the role of virginity in the girls' sexual behavior development. By recognizing the girls' attitudes and beliefs in this regard, we are hoping to provide strategies for designing and implementing sexual health education programs, because studies based on empirical and indigenous research will prove more effective in promoting women's sexual health in societies with similar cultures.

\section{Study aim}

The aim of the present study is development of strategies to promote Iranian girls' sexuality with focus of the role of virginity in the construction of their sexuality.

The specific objectives of this study are to:

1. To determine the girls' perspective about the importance and role of virginity in the construction of the sexuality and its related factors.

2. To compare the girls' perspective born in the 1970s, 80 s and 90 s about the importance and role of virginity in the construction of the sexuality and its related factors.

3. To identify and analyze the girls' interpretations and experiences and its related factors about the impor- 


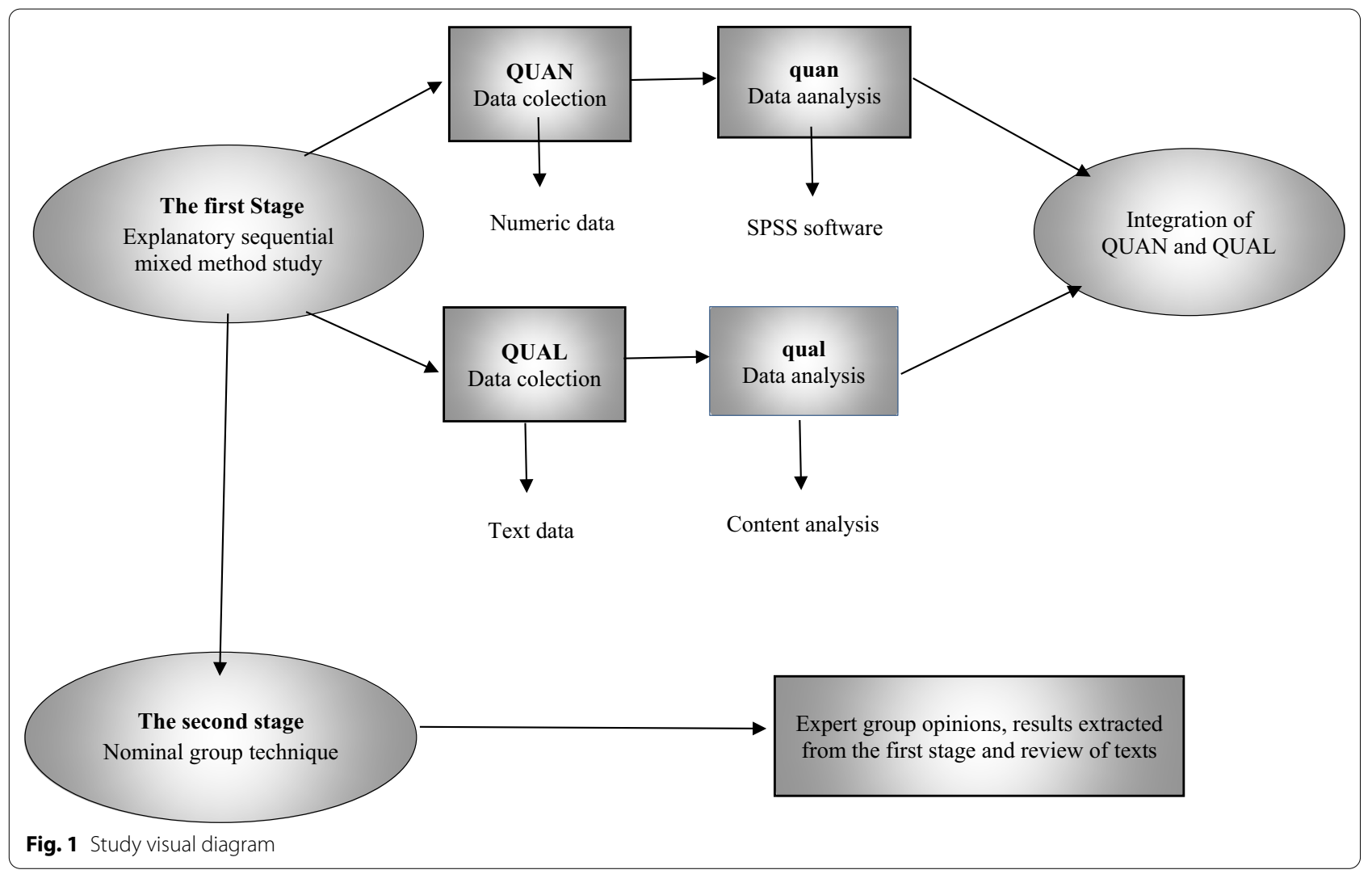

tance of virginity and its role in the construction of the sexuality.

4. To determine strategies to promote healthy sexuality among Iranian girls.

\section{Method}

\section{Study design}

This is a two-stage study; an explanatory sequential mixed-methods follow-up design will be employed in the first stage, which consists of two quantitative and qualitative phases. First, a quantitative phase of the study will be performed, and after the quantitative data are analyzed, the qualitative phase of the study will be performed. In the end of the first stage, quantitative and qualitative data will be combined. In the second phase of this study, a nominal group meeting will be held with the participation of reproductive and sexual health experts (Expert Panel) to develop and present appropriate strategies to promote healthy sexuality in girls, so that the strategies extracted from the results of the first phase and review the texts in this meeting will be provided to experts and after receiving the opinions and ideas of the relevant group of experts in the meeting and prioritizing them, appropriate strategies to improve and promote the healthy sexuality of Iranian girls will be presented. The methodology of the steps is explained below (Fig. 1).

\section{Quantitative study}

The quantitative phase of the study will be based on a cross-sectional population-based survey on the target population of Iranian girls living in Tabriz-Iran (the cohorts of the 1970s, 80s, and 90s).

\section{Sample size and sampling method}

In order to estimate the sample size in the present research, the formula for the estimation of a proportion was employed.

$$
\mathrm{N}=\frac{\left(z_{1}-\frac{\alpha}{2}\right)^{2} P(1-P)}{d^{2}}
$$

In the present study, the z-value was equal to 1.9 at a 95\% confidence level and the p-value based on the study of Honarvar et al. [32] in Iran and considering the d-value was equal to $10 \%$ around $\mathrm{p}$ (proponents of premarital sexual relationships), the sample size was obtained 
459. Due to the use of cluster sampling in this study, the design effect was considered. In most cases, the numerical value of the design effect is considered to be about 1.5-2. It was considered 1.5 in this study and the final sample size was increased to 700 people.

$$
\begin{aligned}
& \mathrm{N}=\frac{(1.96)^{2} 0.456(1-0.456)}{(0.1 \times 0.456)^{2}}=458.3 \cong 459 \\
& \mathrm{~N}=459 \times 1.5=688 \cong 700
\end{aligned}
$$

Sampling in this study is based on population and cluster sampling method, because Tabriz is a large metropolis with different cultures. Attempts will be made in this study to give the whole population of the city a chance to be selected as a sample member. Due to social constraints and sensitivity of the issue of virginity and sexual intercourse and in order to ensure the participants' privacy, the researchers will not interview the participants at their home. Therefore, the research location in this study will be considered health centers. The $1,559,000$ population of Tabriz (according to the 2016 statistics) is distributed in ten urban districts. First, the health centers in Tabriz will be identified and a number of health centers will randomly be selected from each district (There are 83 health centers in Tabriz). The sample size will divided among the selected health centers based on quotas. Households that have a single daughter and meet the inclusion criteria will be randomly selected from among the health centers through the SIB website (This website covers more than 90-92 percent of the population of Tabriz, which is a desirable amount of coverage and the details of Iranian families with all its members are available in the SIB system in health centers and we can access these people). If a family has more than one unmarried girl, all of them will be included in the study if they wish. Then, they will be telephoned and those who are single, were born in the 1970s, 1980s and 1990s and agree to participate in the study, they will be asked how to participate in the study. If they agree and consent, an anonymous email or mobile number will be received from them and they will be sent a questionnaire link to fill out at home under stress-free conditions and in a confidential manner. Otherwise, they will be asked to attend the relevant health center on a specific date to be presented with the questionnaires to complete.

It should be noted that the cohorts of the 1970s, 1980s, and 1990s will be compared as a sub-goal in the quantitative phase of the study. Therefore, we mean the 1970s (born 1971.3.21 to 1981.3.20), the 80s (born 1981.3.21 to 1991.3.20) and the 90s (born 1991.3.21 to 2001.3.20).

\section{Inclusion criteria}

Inclusion criteria in this study are: having Iranian nationality, no history of previous marriage or its contract as defined in the socio-cultural structure of Iran, having or lacking premarrital sex for girls, literacy, born in the 1970s, 1980s and 1990s and having no physical and mental illness as self-report.

\section{Exclusion criteria}

Exclusion criteria are: unwillingness to continue participating in the study, and failure to complete the questionnaire.

\section{Scales and data collection}

The data collection tool in the quantitative stage will be a researcher-made questionnaire to assess the girls' views on virginity and its influential factors. This questionnaire has been designed after reviewing the relevant resources including studies by Carpenter (2002 and 2005) [4, 43], Barnett et al. [44], Eriksson et al. [45], Uecker et al. [46], Wilson et al. [47], Awwad et al. [22], Sprecher et al. [8], Landor et al. [1], Lipman et al. [48], Shirazi et al. [49], Hojat et al. [50], Motamedi et al. [42], Molla et al. [14], Arega et al. [51], Yaşan et al. [52], Teo et al. [20], Khalajabadi-Farahani et al. [31] and Rahmani et al. [53] and tools and based on the specialized experience of the research team. Since virginity and its influential factors are context-based concepts, the research team, having reviewed the existing sources, did not find a tool that socio-culturally suited investigation into these concepts. Therefore, a researcher-made questionnaire will be used to collect information in this phase of the study. The design of this questionnaire will be based on the conceptual framework of the research and its items will correspond to the theory of "social constructivism of sexuality". This questionnaire contains four main parts:

The first part examines the participants' demographic and socio-economic characteristics, including age, education, parental education, current life status, birth location, ethnicity, economic status, communication with parents, and the participants' and their parents' religious affiliation. The items in this part are 16 questions.

The second part is a questionnaire about the girls' individual attitudes regarding the importance of virginity, with items such as "Girls should not have any premarital sexual intercourse", "I believe that boys should remain virgins until marriage because of the importance of cultural and social beliefs", "I preserve my virginity", and "I abstain from any premarital sexual relations due to religious affiliation". This questionnaire will be based on a 5-point Likert scale (totally agree, agree, no opinion, disagree, totally disagree). The items in this part are 12 questions. 
The third part is a questionnaire about the girls' interpersonal attitude questionnaire regarding the importance of virginity, with items such as "I preserve my virginity to respect my family values", "Premarital preservation of virginity is very important for my female friends", and "Boys deceive girls to be their sexual partners by claiming that all the girls have lost their virginity". This questionnaire will be based on a 5-point Likert scale (totally agree, agree, no opinion, disagree, totally disagree). The items in this part are 12 questions.

The fourth part is a questionnaire about the girls' cultural normative attitudes toward the importance of virginity with items such as "In our culture, virginity is particular to girls rather than boys", "In our culture, a virgin bride is preferred", and "Loss of virginity prior to marriage in our culture dishonors the girls". This questionnaire will be based on a 5-point Likert scale (totally agree, agree, no opinion, disagree, totally disagree). The items in this part are seven questions.

The validity and reliability of these questionnaires will be measured before the commencement of sampling. The psychometric properties of the present researcher-made questionnaire will be examined before use in the following way:

Face validity In this study, face validity will be examined both qualitatively and quantitatively. In qualitative face validity, items will be examined in terms of level of difficulty, appropriateness and ambiguity. In quantitative face validity, a Likert scale will be considered for each item of the questionnaire and based on the responses of 10 members of the target group, the impact score of each item will be calculated.

Content validity In this study, content validity will also be examined both qualitatively and quantitatively. To determine the qualitative content validity, the researchers will consult 10 relevant experts regarding the content of the items, the general structure of the questionnaire and any removal or addition of the items (According to statistical tests available for instrument psychometrics, 10 experts are the minimum number in the panel of experts [54]). For quantitative content validity, they will use two indicators of CVR and CVI. In the content validity ratio, the necessity of an item inclusion will be evaluated from the point of view of the experts who respond each item with one of the following three options: (1) necessary (2) useful but unnecessary and (3) unnecessary. To calculate the content validity index, they will have 10 relevant experts evaluate the content validity using the three criteria of simplicity, clarity and specificity or relevance separately based on a 4-point Likert scale.

\section{Data analysis}

The data will be analyzed using SPSS, 24. To describe the demographic and socio-economic characteristics, will be used descriptive statistics including frequency (percentage) and mean (SD) in the case of normal data distribution, and median (quartiles 25-75) in the case of non-normal data distribution. To compare girls' perspective born in the 1970s, 1980s and 1990s about the importance and role of virginity in the construction of the sexuality, in bivariate analysis, one-way analysis of variance and chi-square tests will be used; in multivariate analysis, multivariate linear regression or multivariate logistic regression will be employed by controlling the demographic and socio-economic variables.

\section{Qualitative study}

The data will be analyzed using the conventional content analysis approach [55]. Considering the objectives of the study in its qualitative stage, the use of this method can help the researcher to better understand the situation to explain the role of virginity in the construction of the sexuality among Iranian girls.

\section{Sampling method and data collection}

Participants will be selected through purposive sampling. In the qualitative phase, they will be selected from among the girls who participate in the quantitative phase of the study and are willing to participate in the qualitative phase of the study, and based on either end of the response spectrum or the significance of the relevant factors of the sample. Attempts will be made to have maximum diversity in terms of underlying factors such as education, age, socio-economic status, religion and sexual relations. Interviews will also be conducted in places such as universities, health centers, or in places where participants feel more comfortable and safe. The respondents will be asked through semi-structured indepth interviews, starting with the interview guide and an open-ended question, followed by questions about attitudes towards the importance of virginity and its role in the construction of the sexuality of Iranian girls to reach the qualitative research questions. The interview guide of the quantitative part contains the following questions:

- What individual factors have affected your perspective of virginity? 
- To what extent has religious affiliation affected your perspective of virginity?

- To what extent has the family affected your perspective of virginity?

- To what extent have same- and opposite-sex friends and peers affected your perspective of virginity?

- To what extent has your society affected your perspective of virginity?

- What strategies do you suggest for healthy sexuality promotion in girls?

The research tool in the qualitative part is a researchermade questionnaire. All interviews will be recorded by a digital tape recorder after the participants' consent is obtained. The researcher will also take notes during the interview, recording the participants' responses and facial expressions while they are expressing their specific experiences and views. The duration of the interview will be $45-60 \mathrm{~min}$, which may vary somewhat depending on the experiences of individuals. Also, due to the sensitivity of the research topic and the shame of talking about sexual issues and premarital sex, it will be possible for participants not to tell the truth. The researcher will try to explain the importance of the research topic, establish communication and friendly interaction with the participants and observe the ethical principles in order to gain the trust and confidence of the participants and overcome this limitation.

\section{Data analysis}

Immediately after data collection, they will be analyzed and the coding process began. Qualitative data analysis will be performed using conventional content analysis using the method attributed to Graneheim and Lundman.

Graneheim and Lundman explain, qualitative content analytical approaches focus on analyzing both the explicit or manifest content of a text as well as interpretations of the 'latent content' of texts that which can be interpreted or interpolated from the text, but is not explicitly stated in it [56]. Also conventional content analysis, coding categories are derived directly from the text data. With a directed approach, analysis starts with a theory or relevant research findings as guidance for initial codes. It should be noted that although the questions of the qualitative section are based on the theoretical framework, open codes will be used in data analysis and categories will be extracted from the data based on conceptual commonalities and in response to the research question. Therefore, the type of qualitative study approach will be of the conventional content analysis type [57].
The second stage of research: nominal group technique In the end of the first stage, quantitative and qualitative data will be combined for further explanation and interpretation to be used in the second stage to provide appropriate strategies for the girls' healthy sexuality promotion.

The nominal group technique will be used to develop and present appropriate strategies to promote healthy sexuality in girls. The nominal group technique (NGT) is a structured group-based technique used to build consensus. Participants are asked to individually reflect and to generate ideas based on predetermined, structured questions asked by a facilitator [58].

To do so, first strategies will be extracted based on the results of the first phase as well as reviews of the literature, then a meeting will be held with reproductive and sexual health experts. Inclusion criteria for participants in the nominal technique include: reproductive and sexual health experts, residents of Iran, full acquaintance with Iranian culture and tradition, working in one of the centers related to girls' health. Initially, the experts will be presented with the strategies extracted from the results of the first phase and the review of the texts, and any member of the group can comment on the main research question, and the ideas of the group members will be recorded. Then they are prioritized, and finally, appropriate strategies for improving and promoting the healthy sexuality of Iranian girls will be presented.

\section{Ethics approval and consent to participate}

This study was approved by the ethics committee of the School of Nursing, Midwifery and Rehabilitation of Tehran University of Medical Sciences (ethics code: IR.TUMS.FNM.REC.1400.021). Inform written consent will be received from all participants in both quantitative and qualitative status. The participants will be assured about the confidentiality of information and privacy of their identity. It will also be explained that they are allowed to quit the study at any stage of intervention, and their refusal to cooperate is free at any stage, and no change will be made in presenting or in the quality of services offered to them.

\section{Discussion}

Given that there is a different understanding of the concept of virginity in different parts of the world, so that it is considered a value in some societies but an antisocial behavior in others; it is particular to women and is governed by dual gender standards in some societies, while it is not gender-specific in others. All of these issues can be 
rooted in the religious, social, and cultural structures that dominate different societies [19, 59, 60].

Since the mentioned studies have different cultural, social and religious backgrounds from Iran where few studies have dealt with the concept of virginity only qualitatively $[31,59,61,62]$, it seems that the present study, by employing a better quantitative and qualitative approach, can clarify the young generation's view in the socio-cultural background of Iran and explore the possible inconsistency and contradictions in the modern Iranian society to determine whether the traditional view of the concept of virginity in Iran is still dominant, or it has been changed by factors such as modernization, global communications, influence from advanced Western societies.

This study is one of the few studies that will be conducted in the field of sexual health and culture in Iran and will use a "mixed-methods" approach to determine and explain the role of virginity in the construction of sexuality from the Iranian girls perspective.

We hope that this study can present evidence-based documents from the latest physical, psychological and social developments in young Iranian girls' sexuality and that the presented healthy sexuality promotion strategies, which will be based on Iranian socio-cultural developments, can provide the basic information required for policy-making and planning for young girls' sexual health. It is also hoped that the findings of this study will be useful in culture-based sexuality education and support for reproductive and sexual health care for the young Iranian generation.

\section{Abbreviations}

CVR: Content Validity Ratio; CVI: Content Validity Index; NGT: Nominal group technique; SD: Standard Deviation.

\section{Acknowledgements}

We should thank the Vice-chancellor for Research of Tehran University of Medical Sciences for their financial support for doctoral dissertation protocol in the field of reproductive health.

\section{Authors' contributions}

$\mathrm{SN}, \mathrm{RM}, \mathrm{FKHF}, \mathrm{MM}$ contributed to the design of the protocol. SN and RM contributed to the implementation and analysis plan. SN and RM has written the first draft of this protocol article and all authors have critically read the text and contributed with inputs and revisions. All authors read and approved the final manuscript.

\section{Funding}

This study is funded by Tehran University of Medical Sciences (Grant Number IR.TUMS.FNM.REC.1400.021).

\section{Availability of data and materials}

Not applicable.

\section{Declarations}

Ethics approval and consent to participate

Written informed consent will be obtained from each participant, this protocol has been approved by the Ethics committee of Tehran University of Medical sciences, Tehran, Iran (IR.TUMS.FNM.REC.1400.021).

\section{Consent for publication}

Not applicable.

\section{Competing interests}

The authors declare that they have no competing interests.

\section{Author details}

'Department of Midwifery and Reproductive Health, School of Nursing and Midwifery, Tehran University of Medical Sciences, P.O. Box: 1419733171 , Tehran, Iran. ${ }^{2}$ Nursing and Midwifery Care Research Centre, School of Nursing and Midwifery, Tehran University of Medical Sciences, P.O. Box: 1419733171, Tehran, Iran. ${ }^{3}$ Department of Population \& Health, National Population Studies and Comprehensive Management Institute of Iran, Tehran, Iran. ${ }^{4}$ Department of midwifery, Social Determinants of Health Research Centre, Tabriz University of Medical Sciences, Tabriz, Iran.

Received: 8 June 2021 Accepted: 29 November 2021

Published online: 24 February 2022

\section{References}

1. Landor AM, Simons LG. Why virginity pledges succeed or fail: the moderating effect of religious commitment versus religious participation. J Child Fam Stud. 2014;23(6):1102-13.

2. Ababio P, Yendork JS. "When you talk about virginity it's about females": views on virginity among University of Ghana students. Sex Cult. 2017;21(2):549-68.

3. Kefallinos E. Eternal hymen: Greek-Australian female narratives of virginity in the post-postmodern epoch. Mod Greek Stud (Australia and New Zealand). 2012;15:165-86.

4. Carpenter LM. Gender and the meaning and experience of virginity loss in the contemporary United States. Gend Soc. 2002;16(3):345-65.

5. Erickson PI, Badiane L, Singer M. The social context and meaning of virginity loss among African American and Puerto Rican young adults in Hartford. Med Anthropol Q. 2013;27(3):313-29.

6. Cha ES, Doswell WM, Kim KH, Charron-Prochownik D, Patrick TH. Evaluating the theory of planned behavior to explain intention to engage in premarital sex amongst Korean college students: a questionnaire survey. Int J Nurs Stud. 2007:44(7):1147-57.

7. Halpern CT, Haydon AA. Sexual timetables for oral-genital, vaginal, and anal intercourse: sociodemographic comparisons in a nationally representative sample of adolescents. Am J Public Health. 2012;102(6):1221-8.

8. Sprecher S, Treger S. Virgin college students' reasons for and reactions to their abstinence from sex: results from a 23-year study at a Midwestern US university. J Sex Res. 2015;52(8):936-48.

9. Gesselman AN, Webster GD, Garcia JR. Has virginity lost its virtue? Relationship stigma associated with being a sexually inexperienced adult. J Sex Res. 2017;54(2):202-13.

10. Landor AM, Simons LG. Correlates and predictors of virginity among heterosexual African American young adults. Sex Cult. 2019;23(3):943-61.

11. Cinthio H. "You go home and tell that to my dad!" conflicting claims and understandings on hymen and virginity. Sex Cult. 2015;19(1):172-89.

12. Matswetu VS, Bhana D. Humhandara and hujaya: virginity, culture, and gender inequalities among adolescents in Zimbabwe. SAGE Open. 2018:8(2):1-11.

13. Nkwemu S, Jacobs CH, Mweemba O, Sharma A, Zulu JM. "They say that I have lost my integrity by breaking my virginity": experiences of teen school going mothers in two schools in Lusaka Zambia. BMC Public Health. 2019;19(1):62.

14. Molla M, Berhane Y, Lindtjørn B. Traditional values of virginity and sexual behaviour in rural Ethiopian youth: results from a cross-sectional study. BMC Public Health. 2008;8(1):9. 
15. Mulumeoderhwa M. Virginity requirement versus sexually-active young people: what girls and boys think about virginity in South Kivu, Democratic Republic of Congo. Arch Sex Behav. 2018;47(3):565-75.

16. Nhan NTT, Phu PT, Tra PTT, Phuong PTA, Nhu NQ, Thu NT, et al. Knowledge, attitudes and behaviors of premarital sex among bedsit-living students in Hue City, Vietnam. J Public Health Epidemiol. 2019;11(4):84-9.

17. Dinh $\mathrm{TH}$, Teijlingen ER. Factors influencing engagement in premarital sex among Vietnamese young adults: a qualitative study. Int J Adolesc Med Health. 2019. https://doi.org/10.1515/ijamh-2018-0201.

18. Majumdar C. Attitudes towards premarital sex in India: traditionalism and cultural change. Sex Cult. 2018;22(2):614-31.

19. Manalastas EJ, David CC. Valuation of women's virginity in the Philippines. Asian Women. 2018;34(1):23-48.

20. Teo HSY, Simon A. The perception of premarital sex among students in a religious moral based university. In: Abstract proceedings international scholars conference. 2019;7(1):1558-5.

21. Ayu SM, Sofiana L, Wibowo M, Gustina E, Setiawan A. Predisposing, enabling and reinforcing factors of premarital sex behavior in school adolescents. KEMAS J Kesehat Masy. 2019;15(1):29-38.

22. Awwad J, Nassar A, Usta I, Shaya M, Younes Z, Ghazeeri GH. Attitudes of Lebanese university students towards surgical hymen reconstruction. Arch Sex Behav. 2013;42(8):1627-35.

23. Wynn L, Hassanein S. Hymenoplasty, virginity testing, and the simulacrum of female respectability. Signs. 2017;42(4):893-917.

24. Smith TW, Son J. Trends in public attitudes about sexual morality. NORC at the University of Chicago; 2013.

25. Twenge JM, Sherman RA, Wells BE. Changes in American adults'sexual behavior and attitudes, 1972-2012. Arch Sex Behav. 2015;44(8):2273-85.

26. Rafizadeh M. Sexual politics in modern Iran. Gend Manag. 2011;26(6):451-2.

27. Ghorashi Z. Teenage sexting and sexual behaviors in an Iranian setting. Sex Cult. 2019;23(4):1274-82.

28. Robatjazi M, Simbar M, Nahidi F, Gharehdaghi J, Emamhadi M, Vedadhir AA, Alavimajd H. Virginity testing beyond a medical examination. Glob J Health Sci. 2016;8(7):152.

29. Heydarabadi A, Ramazanpour R, Mojtabavi SS, Firozmand M. Investigating the phenomenon of generation gap and the social factors affecting it between parents and students. J Specialized Soc Sci. 2015;12(15):43-63.

30. Zarean M. Investigation of the reasons for delayed marriage from the viewpoint of female students in Tehran. J Woman Fam Stud. 2018;6(2):86-110.

31. Khalajabadi-Farahani F, Mansson SA, Cleland J. Engage in or refrain from? A qualitative exploration of premarital sexual relations among female college students in Tehran. J Sex Res. 2019:56(8):1009-22.

32. Honarvar B, Salehi F, Barfi R, Asadi Z, Honarvar H, Odoomi N, Arefi N, Bagheri LK. Attitudes toward and experience of singles with premarital sex: a population-based study in Shiraz, southern Iran. Arch Sex Behav. 2016;45(2):395-402

33. Zahedi R, Nasiri N, Zeinali M, Noroozi A, Hajebi A, Haghdoost AA, Pourdamghan N, Sharifi A, Baneshi MR, Sharifi H. The prevalence and associated factors of extra/pre-marital sexual behaviors among University Students in Kerman, Iran. Int J High Risk Behav Addict. 2019;8(1):1-9.

34. Mahmoodi M, Mirzaee M, Jahani Y, McFarland W, Sharifi H. Age and factors associated with first non-marital sex among Iranian youth. Sex Cult. 2019:1(1):1-11.

35. KhalajabadiFarahani F, Cleland J. Perceived norms of premarital heterosexual relationships and sexuality among female college students in Tehran. Cult Health Sex. 2015;17(6):700-17.

36. Rahmani A, Merghati-Khoei E, Moghaddam-Banaem L, Hajizadeh E, Montazeri A. The viewpoints of sexually active single women about premarital sexual relationships: a qualitative study in the Iranian context. Int J High Risk Behav Addict. 2016;5(1):1-6.

37. Robatjazi M, Simbar M, Nahidi F, Gharedaghi J, Vedadhir A, Emamhadi MA, Jafari A. Survey the concepts of virginity \& its pathology. Iran J Forensic Med. 2017;23(1):25-36.

38. Strong B, Cohen TF. The marriage and family experience: intimate relationships in a changing society. Cengage Learn. 2020.

39. AzadArmaki T, Saei MS. Sociological explanation of anomic sexual relationships in Iran. J Fam Res. 2012;7(4):435-62.

40. Ahmadi A. Recreating virginity in Iran: hymenoplasty as a form of resistance. Med Anthropol Q. 2015:30(2):222-37.
41. Noroozi M, Effat-al-Sadat Merghati Khoei F, Taleghani MT, Gholami A. How does a group of Iranian youth conceptualize their risky sexual experiences? Iran Red Crescent Med J. 2015;17(2):2-6.

42. Motamedi M, Merghati-Khoei E, Shahbazi M, Rahimi-Naghani S, Salehi M, Karimi M, Hajebi A, Khalajabadi-Farahani F. Paradoxical attitudes toward premarital dating and sexual encounters in Tehran, Iran: a cross-sectional study. Reprod Health. 2016;13(1):1-10.

43. Carpenter L. Virginity lost: an intimate portrait of first sexual experiences. New York: NYU Press; 2005.

44. Barnett MD, Moore JM. The construct validity of the First Coital Affective Reaction Scale and Virginity Beliefs Scale. Pers Individ Differ. 2017:109:102-10.

45. Eriksson J, Humphreys T. Virginity Beliefs Scale in HANDBOOK of sexualityrelated measures, vol. Fourth edition. New York: Taylor \& Francis group; 2020. p. 92-3.

46. Uecker JE, Angotti N, Regnerus MD. Going most of the way: "Technical virginity" among American adolescents. Soc Sci Res. 2008;37(4):1200-15.

47. Wilson KL, Smith ML, Menn M. Abstinence-related word associations and definitions of abstinence and virginity among Missouri High School Freshmen. J Sch Health. 2013;83(1):1787-94.

48. Lipman CM, Moore AJ. Virginity and guilt differences between men and women. Butler J Undergrad Res. 2016;2(1):116-33.

49. Shirazi KK, Morowatisharifabad MA. Religiosity and determinants of safe sex in Iranian non-medical male students. J Relig Health. 2009;48(1):29-36.

50. Hojat M, Shariat SV, Ansari S. Iranian college students' attitudes toward premarital sex, marriage, and family before and after the Islamic Revolution. Int J Sex Health. 2015;27(3):224-34.

51. Arega WL, Zewale TA, Bogale KA. Premarital sexual practice and associated factors among high school youths in Debretabor town, South Gondar zone, North West Ethiopia, 2017. BMC Res Notes. 2019:12(1):314.

52. Yaşan A, Essizoglu A, Yildirim EA. Predictor factors associated with premarital sexual behaviors among university students in an Islamic culture. Int J Sex Health. 2009;21(3):145-52.

53. Rahmani A, Mezel JA, Allahqoli L, Fallahi A, Sadeghi N, Higgs P. Risky Sexual Behaviour Assessment Scale for Young Women: development and psychometric assessment. Gend Sex Asia Pac. 2019;43:1-11.

54. Munro BH. Statistical methods for health care research, vol. 1. Philadelphia: Lippincott Williams \& Wilkins; 2005

55. Spannagel C, Gläser-Zikuda M, Schroeder U. Application of qualitative content analysis in user-program interaction research. In: Forum Qualitative Sozialforschung/forum: qualitative social research. vol 6, no. 2. 2005.

56. Graneheim UH, Lundman B. Qualitative content analysis in nursing research: concepts, procedures and measures to achieve trustworthiness. Nurse Educ Today. 2004;24(2):105-12.

57. Hsieh HF, Shannon SF. Three approaches to qualitative content analysis. Qual Health Res. 2005;15(9):1277-88.

58. MacPhail A. Nominal group technique: a useful method for working with young people. Br Educ Res J. 2001;27(2):161-70.

59. Rahmani A, Merghati-Khoei E, Moghadam-Banaem L, Hajizadeh E, Hamdieh M, Montazeri A. Development and psychometric evaluation of the Premarital Sexual Behavior Assessment Scale for Young Women (PSASYW): an exploratory mixed method study. Reprod Health. 2014;11(1):1-7.

60. Eşsizoğlu A, Yasan A, Yildirim EA, Gurgen F, Ozkan M. Double standard for traditional value of virginity and premarital sexuality in Turkey: a university students case. Women Health. 2011;51(2):136-50.

61. Kaivanara M. Virginity dilemma: re-creating virginity through hymenoplasty in Iran. Cult Health Sex. 2016;18(1):71-83.

62. Mehrolhassani MH, Yazdi-Feyzabadi V, Mirzaei S, Zolala F, Haghdoost AA, Oroomiei $\mathrm{N}$. The concept of virginity from the perspective of Iranian adolescents: a qualitative study. BMC Public Health. 2020;20:1-8.

\section{Publisher's Note}

Springer Nature remains neutral with regard to jurisdictional claims in published maps and institutional affiliations. 